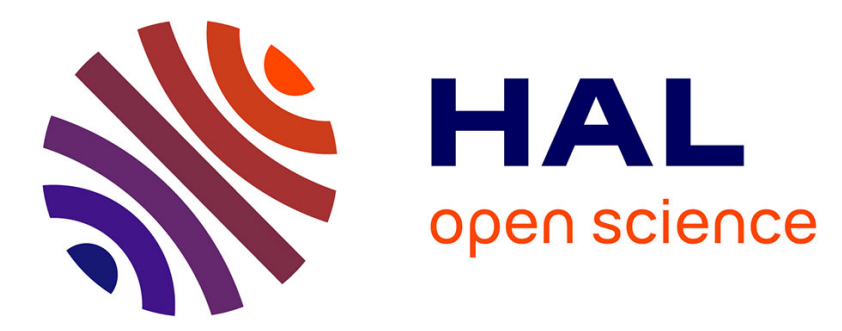

\title{
À plusieurs voix sur Le temps infini des longues peines
} Anne-Marie Marchetti, Roland Pfefferkorn, Nicolas de Laëk, Marc Bessin

\section{To cite this version:}

Anne-Marie Marchetti, Roland Pfefferkorn, Nicolas de Laëk, Marc Bessin. À plusieurs voix sur Le temps infini des longues peines. Mouvements: des idées et des luttes, 2002, L'économie sociale et solidaire, un pojet politique, 19, pp.152-163. hal-01293396

\section{HAL Id: hal-01293396 \\ https://hal.science/hal-01293396}

Submitted on 24 Mar 2016

HAL is a multi-disciplinary open access archive for the deposit and dissemination of scientific research documents, whether they are published or not. The documents may come from teaching and research institutions in France or abroad, or from public or private research centers.
L'archive ouverte pluridisciplinaire HAL, est destinée au dépôt et à la diffusion de documents scientifiques de niveau recherche, publiés ou non, émanant des établissements d'enseignement et de recherche français ou étrangers, des laboratoires publics ou privés. 


\section{À plusieurs voix sur \\ Le temps infini \\ des longues peines.}

Anne-MARIE MARChettI

Perpétuités. Le temps infini des longues peines

Éditions Plon (Terre humaine),

2001,400 p., $22,71 €$

(148,97 FF).
À l'approche des élections, comme prévu, I'horreur carcérale et les défaillances de la politique pénitentiaire, qui avaient mobilisé jusqu'aux parlementaires durant l'année 2000, ont laissé la place, dans les préoccupations médiatiques et politiques, à la thématique de l'insécurité. En témoigne l'évolution de plus en plus sécuritaire du projet de loi pénitentiaire, devenu par exemple silencieux sur les peines incompressibles. Pourtant, l'introduction dans le Code pénal de mesures dites " de sûreté " revient en fait à nier toute possibilité pour un condamné à s'inscrire dans le temps avant dix, vingt ou trente ans. Les détenus condamnés à perpétuité, en particulier, sont ainsi confrontés à une politique d'aménagement des peines qui ne fonctionne quasiment plus. Dès lors, comment font-ils pour survivre et s'inscrire dans un temps infini, parce qu'indéfini ? Le livre d'Anne-Marie Marchetti, issu d'une enquête auprès de perpètes, vient à point nommé rappeler qu'une société qui ne donne pas de perspectives temporelles à ses justiciables, eussent-ils commis les pires crimes, sort du registre de la Justice. Pour présenter cet ouvrage, Roland Pfefferkorn s'entretient avec l'auteure, alors que Nicolas de Laëck, actuellement incarcéré, apporte à propos du livre son témoignage de condamné à perpétuité. Marc Bessin présente quant à lui les intérêts sociologiques et ethnographiques de ce travail. 


\title{
Entretien avec Anne-Marie Marchetti
}

\author{
Anne-Marie Marchetti est sociologue, elle a travaillé \\ pour le CNRS et a publié différentes recherches portant \\ sur l'univers carcéral. Elle enseigne aujourd'hui \\ la sociologie à Amiens, à l'université de Picardie. \\ Elle s'entretient avec Roland Pfefferkorn (université \\ Marc-Bloch, Strasbourg) sur son dernier ouvrage.
}

\begin{abstract}
Mouvements :
Le moins que l'on puisse dire, c'est que Perpétuités. Le temps infini des longues peines ne laisse pas indifférent... Dans le contexte actuel, de plus en plus sécuritaire, comme le montrent bien par exemple les analyses de Loïc Wacquant ou de Laurent Bonneli, son objet ne va pas forcément de soi. S'intéresser à ceux qui ont été condamnés lourdement et qui passent une grande partie de leur vie en prison, les écouter, les comprendre, saisir leur part d'bumanité relève presque du tabou, sinon de la provocation, pour une fraction importante de la population et pour une partie des professionnels de la justice ou du personnel politique qui ont été convertis à la politique de la "tolérance zéro ", qui conduit à la pénalisation de la misère.
\end{abstract}

L'état déplorable des prisons de notre pays et les conditions d'incarcération indignes d'une société véritablement civilisée sont pourtant dans le même temps, ces dernières années, dénoncés régulièrement dans la presse et dans d'autres médias. Parfois même avec une force de conviction étonnante. On se souvient du livre $d u$ médecin-chef de la prison de la Santé qui avait fait quelques grosses vagues. Il a même conduit à la mise en place d'une commission d'enquête parlementaire qui n'a pu que corroborer ces constats accablants. On connaît aussi l'action de veille ou de surveillance citoyenne, si on peut se permettre une telle expression, mise en ouvre par l'Observatoire international des prisons. Cette ONG est particulièrement attentive à ce que les prisonniers n'endurent pas des peines supplémentaires (notamment pour tout ce qui concerne l'bumiliation, les agressions, la pauvreté, etc.) qui s'ajoutent trop souvent à la privation de liberté à laquelle ils ont été condamnés.

Les établissements pour peine doivent pourtant, du moins en théorie, contribuer à ce que les prisonniers sortent dans de bonnes conditions, c'est-à-dire qu'ils puissent se réinsérer de façon satisfaisante, à la fois pour eux-mêmes et leurs proches quand ils en ont, et dans l'intérêt de l'ensemble de la société... Or, le livre montre que les conditions actuelles de l'incarcération, et plus particulièrement la durée effective des peines, contribuent trop souvent à déshumaniser ceux qui sont condamnés par les tribunaux à passer une longue, parfois très longue, partie de leur vie en prison. Les "longues peines" sont de plus en plus nombreux car la durée des 
peines tend très nettement à s'allonger ces dernières années. 14,4\% des peines dépassaient dix ans au $1^{\text {er }}$ janvier 1994, en 2000 la proportion est passée à $21,4 \%$.

L'enquête est en premier lieu un témoignage implacable sur la vie dans les établissements pour peine, un témoignage à la fois rigoureux et sensible qui donne à entendre la parole de ces prisonniers, dans toute leur diversité, des bommes, des femmes, des gens venant le plus souvent des catégories sociales les plus modestes. Il montre en même temps très clairement que les prisonniers ne se réduisent pas à des modèles typiques, à des abstractions, à des variables sociologiques. Les prisonniers dont il rapporte la parole se livrent dans leur singularité.

Le lecteur peut difficilement rester impassible face au contenu du livre. D'autant que ce dernier mêle, de manière particulièrement beureuse, différents registres d'écriture. La vie même se donne à lire dans le texte, vie mutilée souvent, vie désespérée et desséchée parfois, vie en attente toujours. Les mots des emprisonnés, leur parole, sont présents tout au long des quelque trente chapitres du livre.

C'est en second lieu une analyse sociologique précise et détaillée de la vie carcérale des personnes concernées par les longues peines. Et c'est en même temps un compte-rendu d'enquête qui n'occulte pas les conditions de réalisation de ce travail, ni les effets de l'enquête sur la sociologue. Les carnets d'enquête insérés au fur et à mesure dans le texte montrent qu'on ne sort pas indemne d'une telle investigation.

Ce livre est enfin, c'est suffisamment rare pour être souligné, une magnifique ouvre lit- téraire qui fait bonneur à la collection qui l'accueille (Terre Humaine, dirigée par Jean Malaurie) et qui montre que sociologie et littérature ne sont pas forcément antinomiques. Ma première question porte sur votre intérêt pour les prisons et ceux qui y vivent. Comment en êtes-vous arrivée à vous préoccuper plus particulièrement des personnes condamnées à de longues peines?

Anne-Marie Marchetti : Mon intérêt pour les " longues peines " date de 1993. À l'époque je réalisais une enquête commanditée par le ministère de la Justice sur la pauvreté en prison, et parmi les pauvres qu'on me faisait rencontrer en détention, il $\mathrm{y}$ avait des perpètes. Je me suis rendu compte que plusieurs d'entre eux étaient désignés comme pauvres non pas parce qu'ils l'étaient financièrement, mais parce que les travailleurs sociaux les trouvaient carencés dans pas mal de domaines : c'étaient des " pauvres gars " en quelque sorte. Or, au même moment Pierre Méhaignerie ${ }^{1}$ proposait le projet de loi dit de perpétuité réelle. Cela m'a interpellée ; je me suis demandé si l'on se rendait bien compte de ce que l'on faisait et de ce que l'on allait faire vivre à ces hommes, à supposer que l'on s'en soucie ! Je me suis aussi demandé jusqu'à quand, dans le contexte carcéral, un être humain pouvait évoluer positivement et je me suis promis d'enquêter un jour là-dessus.

$\mathbf{M}$ : En ouverture à votre livre, vous citez Hegel : "La pensée abstraite ne voit dans l'assassin rien d'autre que cette qualité abstraite et détruit en lui, à l'aide de cette seule qualité, tout le reste de son humanité".

1. Alors ministre de la Justice du gouvernement Balladur.
N'est-on pas resté très largement, dans le sens commun, notamment dans trop de discours relayés par les médias, au niveau de cette pensée abstraite?

A.-M. M. : Oui, je trouve une partie du discours véhiculé par les médias - du moins certains souvent effrayant, car il est réducteur. Cela dit, il reflète parfois celui de certains magistrats. Lors d'un récent procès, un avocat général a présenté l'accusé comme étant " l'incarnation du diable ". Que quelqu'un qui est censé représenter le point de vue de la société tienne des propos de cet acabit est consternant. Présenter un meurtrier comme le diable, un monstre, une bête fauve, etc. (propos fréquemment entendus lors des assises) est grave. Cela déshumanise le coupable, vide de son sens le procès (si ce n'est pas le procès d'un " humain " qui se fait, à quoi rime donc cette procédure ?) et rend la sentence presque inacceptable pour les condamnés puisqu'ils se sentent rejetés du corps social. Il faut raison garder. Ce n'est pas parce que l'on est capable du pire, que l'on n'est capable que du pire. Les êtres humains ont de multiples facettes et il est aussi inhumain de réduire un criminel à son crime que de réduire un cancéreux à son cancer. L'Homme vaut toujours mieux que ses actes.

M : Vous avez cherché à aller au-delà de cette "pensée abstraite ", dénoncée en son temps par Hegel, en tentant de comprendre ce que vivent les prisonniers condamnés à de longues peines. Pouvez-vous vous expliquer sur ce point?

A.-M. M. : J'ai surtout cherché à comprendre comment ils organisaient leur survie. Survie à la peine et survie au crime dans un univers qui est particulière- 
ment dur, l'univers carcéral. Cela implique pour beaucoup d'aller chercher de la force au plus profond de soi tout en négociant avec les contraintes du milieu ambiant. Ma recherche s'apparente de ce point de vue à celle de Bettelheim. Elle aurait pu s'appeler elle aussi " survivre " d'autant plus que les analogies entre les modes de fonctionnement qu'adoptent les déportés et les " longues peines " sont nombreuses.

$\mathbf{M}$ : La première partie de votre livre s'intitule : Le temps des maisons d'arrêt : survivre à l'insupportable ». En quoi la vie dans ces prisons est-elle si insupportable?

Quand je parle de "survivre à l'insupportable ", je ne fais pas uniquement allusion à la prison, même si elle fait aussi partie de l'insupportable. Pour me faire mieux comprendre, je vais donner l'exemple d'une femme qui a assassiné son enfant. Une fois incarcérée, elle doit faire face aux réactions de ses codétenues face à son crime. Ça se passe souvent très mal : cette femme sera molestée, se mettra à vivre confinée dans sa cellule ; sa famille va la rejeter, ses gosses vont souvent être placés à l'ASE. Bref, son crime va entraîner toute une série de ruptures et de pertes, sans compter la perspective de vivre des années de détention dans un climat de violence et d'hostilité. De surcrôt, contrairement à ce qu'on laisse souvent accroire, les assassins se trouvent parfois endeuillés par le crime commis d'autant plus que la victime est souvent un proche (objet de haine et d'amour). On peut à la fois se sentir soulagé par un crime qui met fin à une souffrance et se sentir aussi très mal de ce que l'on a fait. Et puis les maisons d'arrêt où les meurtriers passent leurs premières années de détention cumulent pour la plupart les principales carences des établissements pénitentiaires : vétusté, promiscuité, pénurie d'emploi, racket, etc. Cette temporalité est donc particulièrement difficile à vivre, ce qui explique d'ailleurs l'importance des suicides pendant cette période.

$\mathbf{M}$ : Est-il possible de caractériser, dans les grandes lignes au moins, le quotidien d'un condamné à de longues peines, si tant est qu'il n'existerait qu'un seul type de condamnés à de longues peines?

A.-M. M. : Il est difficile de répondre à cette question d'une façon univoque parce qu'il y a plusieurs types de condamnés (auteurs de crimes stigmatisés ou non, malades mentaux ou gens " normaux ", etc.) et que la vie n'est de toute façon pas la même en maison d'arrêt quand on se retrouve confiné en cellule vingt et une heures par jour avec des codétenus et quand on est seul en établissement pour peine (c'est-à-dire le type de structure où sont envoyés les condamnés à des peines relativement longues). Dans ce type d'institution l'autonomie dont jouissent les détenus est généralement un peu plus grande et le spectre d'activités auxquelles ils peuvent se livrer, plus large. Cela dit, le quotidien

\section{Certains tiendront}

\section{dix ans, quinze ans et \\ puis un jour après un}

refus de conditionnelle,

\section{ils laisseront tout}

tomber, aller à vau-

I'eau. Ils seront finis. des " longues peines " rappelle souvent celui des maisons de retraites : même monotonie, faible possibilité de rencontres, gamme de plaisirs limitée et solitude fréquente. Une vie en quelque sorte émoussée.

$\mathbf{M}$ : Nous savons tous que tous les êtres bumains sont d'une certaine manière prisonniers du temps. Les condamnés à des longues peines, dix, vingt, trente années d'enfermement, le savent probablement davantage encore. Comment ces prisonniers "s'arrangent-ils" avec le temps, le temps de la prison, le temps qui passe, le temps de la vie qui passe?

A.-M. M. : Certains ne s'en arrangent pas. Ils se suicident (la France est numéro un en Europe dans ce domaine) ou s'évadent (mais les cavales se terminent en général mal), ou deviennent de plus en plus fous, ou se dessèchent comme certains vieux. Certains font avec parce que leur vie avant la prison était déjà extrêmement morne (mais peut-être se blindent-ils un peu plus); d'autres trouvent suffisamment de ressources en eux-mêmes et autour d'eux pour élaborer des projets à court terme d'abord : passer son certif, par exemple, et à long terme bien plus tard. D'autres tiennent grâce à la haine contre les institutions, les jurés, la justice, etc. "On ne les aura pas, on serait trop content!", mais la haine peut aussi rendre fou, faire suffoquer, débloquer. La haine est à géométrie variable suivant la temporalité où l'on se place ; certains tiendront dix ans, quinze ans et puis un jour après un refus de conditionnelle, ils laisseront tout tomber, aller à vau-l'eau. Ils seront finis.

$\mathbf{M}$ : Comment certains prisonniers arrivent-ils à tenir sans 
être irrémédiablement cassés? Quelles sont les caractéristiques de ceux qui tiennent? Quelles sont leurs ressources spécifiques?

A.-M. M. : Même si certaines personnes ne sortent pas irrémédiablement cassées, je pense que l'on doit rarement sortir d'une très longue peine sans "fêlure ". Un directeur d'établissement m'a dit : "On ne sort pas indemne d'un tel parcours ". Ceux qui ne sont pas " cassés " étaient des gens bien structurés psychologiquement avant la détention, ayant des ressources intimes qui leur permettaient de prendre leurs distances par rapport aux épreuves, de se "désidentifier" de l'image du criminel, du détenu, du pauvre type qu'on leur renvoie souvent en détention. Ceux qui tiennent à garder une certaine estime d'euxmêmes, à se trouver des sources de plaisir en détention, à avoir un réseau minimum à l'extérieur, à croire malgré tout en un avenir post-carcéral (c'est plus facile quand on est jeune).

M : Vous montrez dans votre livre que les détenus condamnés à de longues peines qui craquent sont relativement nombreux. Quelles sont leurs caractéristiques? Quels sont les principaux éléments qui leur manquent?

A.-M. M. : Ceux qui craquent sont généralement des gens dont la prison amplifie encore plus les difficultés psychiques, relationnelles, sexuelles et/ou qui n'arrivent pas à faire le deuil de la liberté. J'ai d'ailleurs l'habitude de dire en guise de boutade qu'il ne faudrait mettre en prison que les gens très équilibrés car pour les autres une longue détention est destructrice. Dans la réalité, on fait le contraire : plus les gens sont déséquilibrés, plus ils écope-

\section{Tendanciellement,}

la détention - à crime

égal - des plus pauvres

est beaucoup plus

\section{longue que celle des}

gens plus " fortunés ".

ront de longues peines. Cela dit, on voit aussi fréquemment parmi les "longues peines " des gens qui tiennent puis craquent. On voit beaucoup de hauts et de bas dans les trajectoires carcérales.

M : Avez-vous observé des différences fondamentales parmi les prisonniers selon leur sexe, ou selon leur âge?

A.-M. M. : Parlons d'abord de l'âge ! Je crois qu'il est plus facile de tenir malgré tout quand on est jeune que quand on prend perpète à cinquante ans. L'idée de finir ses jours en prison est désespérante ; ça veut dire finir ses jours sans tendresse, sans accompagnement, en restant un soushomme ad vitam aeternam. Terrible perspective. Et puis les "vieux " sont souvent plus isolés (pas de maman pour les soutenir dehors), plus démunis (moins de possibilités de trouver du travail) et être malade en prison, ce n'est pas simple! Les femmes aussi sont généralement moins soutenues par leurs proches que les hommes (enfants mis à part). Les hommes se trouvent parfois des femmes charmantes et libres par le biais des petites annonces alors que les détenues que j'ai rencontrées cor- respondaient surtout avec des... détenus. Les visites masculines sont plus rares côté femmes que le contraire côté hommes. Avec les femmes, un des gros problèmes de surcrôit c'est de gérer sur le long terme la séparation d'avec les enfants. J'ai connu une femme qui avait pris perpète alors qu'elle était enceinte. Quand elle sortira son enfant sera devenu un adulte. Cette femme avait tué un de ses enfants, mais elle avait aussi le sentiment d'avoir perdu l'autre.

\section{M : Les inégalités sociales face} à la justice pénale sont connues. Avez-vous observé, durant votre enquête, des différences importantes parmi les prisonniers selon les milieux sociaux?

A.-M. M. : La différence entre les milieux sociaux est, elle aussi, fondamentale car la prison est profondément inégalitaire. Les gens qui s'en sortent le mieux d'un point de vue économique, ou dans d'autres domaines, étaient généralement des gens qui avaient quelques " capitaux ": culturel, relationnel, voire économique extra-muros. Les gens aisés toutes choses étant égales par ailleurs - sont aussi ceux qui arrivent le mieux à se projeter dans le temps. La capacité de prévoir l'avenir se trouve difficilement chez des gens qui n'avaient pas d'avenir dehors, qui par exemple étaient précarisés. Le problème, c'est que s'ils ne font que survivre au jour le jour, ils vont le payer. Tendanciellement, la détention - à crime égal - des plus pauvres est beaucoup plus longue que celle des gens plus "fortunés ". Ils accèdent beaucoup plus difficilement aux aménagements de peines que leurs codétenus car ils n'arrivent pas à planifier leur vie et à 
choisir des activités " payantes " (psychothérapie, études, etc.). On vérifie une fois de plus que la prison n'est pas " républicaine".

M : Comment est vécue la sexualité en prison? Comment s'est passée votre confrontation en tant que femme sociologue avec des bommes prisonniers, coupés de tout contact physique, et même souvent de tout contact épistolier, avec des femmes?

A.-M. M. : J'ai du mal à parler de la sexualité en prison car l'attitude des pouvoirs publics à cet égard, et donc la nôtre en tant qu'électeurs, me paraît proprement scandaleuse. Interdire ad vitam aeternam les relations hétérosexuelles en prison (les relations homos sont tolérées, même si ça ne se dit pas) ne se justifie en aucune façon ; c'est absolument indigne d'une démocratie et contraire à toutes les missions qui sont assignées à l'administration pénitentiaire : la réinsertion notamment, et le soi-disant maintien des liens familiaux. Dans la pratique, la sexualité en prison est souvent de l'autoérotisme alimenté par les vidéos porno des circuits internes aux établissements, de l'homosexualité clandestine ou pas (notamment chez les femmes et dans les établissements où l'on trouve le plus de délinquants sexuels) et de la prostitution (les prostitués étant comme dehors des personnes particulièrement vulnérables). Le fait d'être une enquêtricefemme n'est bien sûr pas neutre - mais ça ne l'est de toute façon jamais. J'ai été vue parfois comme une représentante du sexe manquant. On suscite forcément toutes sortes d'attentes, d'espoirs, de confidences et il est bien évident que ça m'a touchée, émue, parfois flattée ou fait peur. J'en ai parlé dans mon
Le fait d'être une enquêtrice-femme n'est bien sûr pas neutre mais ça ne l'est de toute façon jamais. J'ai été vue parfois comme une représentante

du sexe manquant.

livre même si je pense que je n'ai pas complètement épuisé le sujet. Par exemple, face à un violeur, on peut pour une fois ressentir l'inversion du rapport de forces et y trouver fugacement de la jouissance.

$\mathbf{M}$ : Pour clore cet entretien, il est inévitable d'aborder la question des transformations à mettre en ouvre afin d'éviter que la prison ne se réduise, notamment pour les longues peines, à un lieu où les individualités sont écrasées, voire mutilées à jamais. Pour le dire autrement, quelles sont les principales évolutions que vous pensez soubaitables pour contribuer à " bumaniser »la prison? Mais peut-on bumaniser la prison?

A.-M. M. : Avant toute chose selon moi, il faut revoir le Code pénal. Je vais dire quelque chose qui va faire bondir plus d'une personne : nous avons des peines trop longues. Dans une démocratie, gouvernée par des partis de gauche, nous avons en fait une pénalité très répressive qui s'est encore durcie dans le nouveau Code pénal, ce qui pour moi - et Montesquieu ! - caractérise normalement les régimes totalitaires. Ceux qui ont voté les périodes de sûreté de dix-huit à trente ans ont voté des lois scélérates qui nous déshonorent. Certes, l'on doit mettre hors d'état de nuire des gens qui ont commis des atteintes aux personnes, mais il faut aussi que les sanctions aient un sens pour eux. Il faut donc que les prisons soient des lieux de vie et non de survie, que l'on propose aux détenus des prestations de qualité et qu'on bannisse autant que possible des établissements pénitentiaires des interdictions qui n'ont qu'un fondement punitif (absence de relations sexuelles, par exemple). Des sentences plus courtes, plus appropriées il y a trop de malades mentaux en prison - mais des détentions plus sensées, c'est ce vers quoi nous devons tendre. Cela dit, je ne crois pas qu'il y ait quelque part au monde une bonne prison, même si on peut humaniser l'institution. Le danger actuel, en France, c'est que l'on risque de nous proposer des prisons moins choquantes, mais avec des détentions toujours démesurées. C'est ce que semble nous préparer la Chancellerie. Et ça, c'est très insuffisant. D'ailleurs si nous laissons les longues peines s'accroître de plus en plus comme c'est le cas actuellement, nous allons vers des mutineries. Ca me paraît inévitable. Je finirais par une note cynique : je crois que dans l'état actuel des rapports sociaux en France, nous ne réglerons pas au mieux, ni la question des longues peines, ni celle des prisons car les personnes que ces problèmes concernent sont surtout des pauvres gens, et qu'ils n'ont pas suffisamment de poids pour que l'on gère au mieux leurs intérêts.

ENTRETIEN RÉALISÉ PAR RolANd PFEFFERKoRN 


\section{La lecture-témoignage d'un condamné à perpétuité}

$\mathbf{U}$ n ouvrage tel que celui écrit par Anne-Marie Marchetti constitue une nouveauté très importante : celle de la prise en compte d'un vécu particulier, celui des personnes condamnées à perpétuité. La gestion d'un quotidien et d'un environnement délétères, d'une temporalité inaccessible, qui fait du "perpète " un être sans repère et donc a priori sans but, tout cela ne peut et ne doit être pris à la légère. Il en va en effet tant de la santé physique et mentale des détenus que de celle de la société qui fait le choix de les enfermer.

Depuis quelques temps, et indépendamment de la sortie début 2000 de l'ouvrage du docteur Vasseur, le regard porté sur les prisons et leurs " locataires " tend à évoluer. Il faut dire que les années quatrevingt-dix auront constitué aux yeux de nombreux détenus une période critique, mais aussi critiquable. L'aggravation considérable des peines judiciaires prononcées, en tant que réponses fournies à la médiatisation tous azimuts d'affaires criminelles sorties de leur contexte, a accentué le sentiment d'insécurité que certains politiques et médias se sont efforcés de mettre en avant. Si notre société semble effectivement de plus en plus malade de ses propres insatisfactions, elle n'en continue pas moins de chercher ses boucs émissaires, ceux dont la faute impardonnable - la déviance, voire le crime - doit stigmatiser toute leur existence : les longues peines, et tout particulièrement les condamnés à perpétuité dits " perpètes ".

En tant que dernier refouloir des fantasmes de certains, la prison constitue pour autant un monde dont la plupart de ses occupants - pour ne pas dire tous - doivent ressortir un jour. Deux questions se posent alors : quand? dans quel état? Si la peine privative de liberté est un choix sociétal sur lequel je ne reviendrai pas, la question du sens que l'on doit donner à cette peine est inévitable!

Étant moi-même condamné à une peine de perpétuité, et après bientôt treize années d'incarcération, la lecture de cet ouvrage ne fut pas facile. Peut-être parce que, pour la première fois, j'ai trouvé tout au long de ces pages un langage que je connaissais et dans lequel je me retrouvais. Loin de nombreux récits de détenus évoquant leurs parcours et expériences individuelles, loin des classiques ouvrages de recherche souvent centrés sur telle ou telle thématique carcérale, le livre d'Anne-Marie Marchetti fournit un nouveau regard sur les détenus condamnés à de lourdes peines, et ce sans concession aucune pour qui que ce soit. Loin d'un misérabilisme de mauvais goût ou d'une empathie déplacée, elle a su décrire, chez ceux dont la faute ne pourra jamais être effacée, la part d'humanité 
qu'un crime, un procès et un parcours carcéral aurait dû ou pu mettre à néant.

Perpétuité, condamnation à vie, peine perpétuelle... tous ces mots renvoient malgré tout à une réalité : le détenu doit pouvoir un jour être libéré. À moins que la mort ne le rattrape. Si l'idée de la justice-vengeance conserve ses adeptes, la société doit pourtant se faire à l'idée qu'un jour chacun d'entre nous redeviendra libre. À cet égard, la condamnation doit pouvoir aussi être assimilée à un contrat moral dans lequel les deux parties ont leur rôle à jouer : le détenu mais aussi la société à travers l'administration pénitentiaire. Mettre une personne en prison pendant de longues années implique dès lors des obligations pour tout le monde : obligations pour le détenu qui doit pouvoir mettre tout en ouvre pour s'en sortir ; obligations pour l'administration pénitentiaire qui doit pouvoir lui en donner les moyens. Force est de constater que ni l'une ni l'autre ne se font simultanément, et que bien souvent les échecs de l'un sont imputables à l'autre.

Comme beaucoup de "perpètes ", le prononcé du verdict constitue un moment bizarre, irréel et il aura fallu de nombreux jours pour en mesurer toute la portée. Comme beaucoup de criminels " non professionnels ", le procès aura aussi été pour moi ce moment charnière, celui qui aura permis de mesurer l'ensemble des actes commis, de prendre conscience qu'un crime avait eu lieu, qu'une victime avait souffert. Mon expérience n'a rien de typique. Elle est commune à de nombreux " perpètes " croisés au cours de mes années d'incarcération. Elle est surtout commune à de nombreuses personnes condamnées pour crime. Face à cela, le déni est bien souvent la seule réponse immédiate à l'incompréhension, à sa propre incompréhension. Or, cette compréhension est nécessaire, obligatoire si un détenu veut pouvoir un jour se regarder en face, s'assumer, mais aussi regarder son passé et l'affronter. Se positionner par rapport à son crime et surtout à

\section{Si l'idée de la justice-}

vengeance conserve

ses adeptes, la société

doit pourtant se faire

à l'idée qu'un jour

chacun d'entre nous

redeviendra libre.

la victime n'est pas évident, et surtout irréalisable dans un premier temps alors même que tout un appareil judiciaire vous le demande. Cela relève d'un travail intérieur considérable et il peut arriver que ce travail ne se fasse pas. Mais il est nécessaire si l'on veut pouvoir un jour s'en sortir. Le problème du déni de l'acte criminel - qui ne doit en aucun cas être assimilé à de la désinvolture voire du cynisme - est ainsi un élément crucial d'autoprotection. Dans son ouvrage, Anne-Marie Marchetti avait résumé cet aspect par cette simple exclamation : " suis-je cela ? "Répondre de suite par l'affirmative, c'est ouvrir la porte à sa propre perte. Répondre par la néga- tive, c'est peut-être le seul moyen de pouvoir un jour s'en sortir. C'est plus tard, et seulement lorsque tout un travail accompagné ou non - aura été fait que le masque pourra tomber, que les repères pourront (ré)apparaître. Viendra alors le temps où la pleine réalité de ses propres actes et de la souffrance causée pourront s'imposer à soi, et faire ainsi de ce néant une marche vers la lumière.

La vie d'une personne condamnée à perpétuité ne diffère en apparence guère de celle de n'importe quelle autre personne incarcérée. Elle habite les mêmes lieux, côtoie les mêmes personnes, participe au même quotidien. La seule différence est relative au temps. Un temps que l'on ne peut gérer de la même façon et pourtant... Quelle population hétérogène que celle des "perpètes " ! C'est en effet dans cette catégorie de détenus que l'on trouve sûrement la plus grande diversité comportementale. La distinction opérée par Anne-Marie Marchetti entre "végétatifs " et " hyperactifs ", si elle peut paraitre au premier abord manichéenne, renvoie à une situation de fait dont le caractère extrême se rencontre moins souvent que dans d'autres catégories de détenus : comment en effet expliquer que certains se laissent aller jusque dans leur corps et leur âme alors même qu'ils sont en début de peine, alors que d'autres, même après plus de quinze années de détention, soient des plus combatifs ! Lorsque je me trouvais dans un centre de détention du Sud de la France, la plupart des activités culturelles étaient gérées par des "perpètes ". Sans eux, peu d'activités auraient tenu sur la durée. Si la capacité d'initiative de certains avait pu faire 
craindre le pire à une direction souvent réfractaire à ce que de longues peines soient trop autonomes, force est de constater que leur investissement personnel fut à la hauteur. Comment alors expliquer un tel investissement? La volonté de tenir le coup, de s'investir dans du productif alors même que la prison n'offre la plupart du temps que des ersatz d'activités, mais peut-être aussi se prouver à soi - et à une administration souvent encline à vous rabaisser - que l'on vaut encore quelque chose. Malgré tout. En effet, lorsque la perspective d'être enfermé pendant près de vingt années devient réalité, nombre de perpètes auront pu avoir comme premier réflexe celui de se laisser aller, de suivre des événements imposés d'en haut, de "faire sa peine "tout en s'oubliant petit à petit. Par sa violence symbolique, par l'ingestion d'une condamnation déroutante, le procès devient pour certains un détonateur. Pour d'autres, un couperet. Toutefois, du temps est encore bien souvent nécessaire pour affronter cette réalité, mi-temps qui doit s'inscrire dans une temporalité plus grande, celle de la perpétuité. Contrairement à de nombreux détenus condamnés à de lourdes peines, je ne m'inscrivais pas dans un parcours de désocialisation. Criminel à vingt et un ans, j'étais étudiant en faculté. Mais comme beaucoup de mes codétenus, je me sentais perdu, loin de moi et des autres. Condamné à l'âge de vingt-trois ans, ce sont les études universitaires qui m'auront, entre autres, permis de me retrouver. Parce qu'aussi des personnes auront su trouver les mots et le moment opportun pour me rassurer, pour me rendre espoir. Mais aussi me faire confiance. Car on ne vit pas en prison sans les autres, sans la main secourable, d'où qu'elle vienne. Il est cependant souvent difficile de la reconnaître au milieu de toutes les autres, celles qui vous montrent du doigt, celles qui vous jugent et condamnent, et celles qui vous disent de vous taire. Beaucoup de détenus n'ont pu ou su la reconnaître, d'autres se sont trompés, d'autres encore ont été trom-

\section{La volonté de tenir}

\section{le coup, de s'investir}

\section{dans du productif alors}

même que la prison

$$
\text { n'offre la plupart du }
$$

temps que des ersatz

d'activités.

pés. J'ai eu cette chance d'en reconnaître certaines, et surtout de les écouter. Seul, un détenu n'est rien et peu d'espoir lui est permis. Difficile dès lors de donner sa confiance dans un univers où la méfiance est de mise, où les tromperies sont multiples et où le désespoir guette en permanence. Comme d'autres détenus, et parce que mon âge et surtout mon parcours antérieur me le permettaient, j'ai repris des études en prison. Alors que je m'étais attaché dans un premier temps à me reconstruire intellectuellement et psychologiquement, la confiance et le soutien de professeurs d'université m'ont permis d'envisager une suite. La rencontre avec un maître de conférence en licence de socio- logie fut à cet égard décisive. Me proposant de travailler sur la santé en prison - à la veille de l'application de la nouvelle réforme relative à la prise en charge médicale des détenus ma première réaction fut évidemment un refus. Pourquoi en effet faire un travail de recherche sur la prison alors que j'y suis, que ce milieu m'envahit chaque jour un peu plus, me brûle ? Pourquoi m'imposer des souffrances supplémentaires alors que chaque jour passé est un jour à oublier? Par ailleurs, tout s'y opposait : le silence des uns, le mensonge des autres, le refus probable d'une administration peu encline à ce que l'un de ses administrés mette son nez dedans ! Pourtant, l'idée a fait son chemin. Comment dès lors expliquer ce revirement? Plusieurs raisons à cela. La première, et non des moindres, est que dans un milieu de contrainte quasi totale, l'idée de faire un travail interdit m'a attiré. L'interdit par excellence : le détenu qui travaille dans et sur la prison. La deuxième raison, guère différente de la précédente, profondément narcissique et pourtant si bénéfique, était d'aller au bout du travail le plus irréel qui soit pour un détenu, de se prouver que l'on pouvait aller au bout de ce challenge, de ce pari sur soi, sur sa propre destinée. Mais la dernière et véritable raison est venue plus tard, lorsque ce travail avait commencé à prendre forme. Ce fut en effet en travaillant sur et dans la prison, avec des détenus et sur leur propre vie que je pus mieux comprendre la mienne : comprendre mon passé, mes actes mais aussi me regarder enfin en face, ainsi que mon avenir. Il m'était apparu que si le temps infini de la prison pouvait être destruc- 
teur, il allait me donner les moyens de mieux me connaître. C'est ainsi qu'en travaillant sur les souffrances des autres, les miennes et celles que j'avais pu causer, c'était une vie bancale que je pouvais espérer reconstruire.

Ainsi que le rappelle AnneMarie Marchetti, l'administration pénitentiaire a mis en place il y a quelques années ce qu'on appelle le PEP ou Projet d'exécution de peine, qui permettait à chaque détenu de s'inscrire dans un parcours de reconstruction tant intellectuelle que psychologique, voire professionnelle. Pourtant, un mot apparaît ici dérisoire : celui de "projet ". Que signifie dès lors faire des projets en prison? Une question qui peut paraître saugrenue tant cela dépend du détenu que de l'institution ellemême. En effet, la vie des détenus est caractérisée par une assistance à tous les niveaux. Or, sauf à contourner un système qui de toute façon s'impose à vous et vous rattrapera un jour ou l'autre, rien ne peut se faire sans l'aval tacite ou officiel de l'institution et tout particulièrement de la direction de l'établissement où l'on est incarcéré. C'est ainsi qu'ayant dû quitter un précédent établissement pénitentiaire dont le nouveau directeur avait exprimé son refus à ce que je continue ce travail de recherche, j'ai pu intégrer un autre établissement où l'accueil fut plus favorable. Comment expliquer de telles différences de traitement si ce n'est par la diversité des conceptions quant au sens même de la prison et de la peine? Un sujet que certains auront jugé "scabreux ", et d'autres "intéressant ". Un sujet certes délicat mais pourtant d'actualité : les conduites et stratégies de soins des détenus. Comment alors réagir face à cela, parce qu'un nouveau directeur n'avait pas les mêmes vues que son autorité de tutelle ou son prédécesseur ? Comment affronter un tel retournement, un tel refus sans fondement quand il succède à plusieurs années d'investissement personnel ? Comment affronter psychologiquement un mur que l'on érige devant un horizon déjà largement obstrué ?

Si la violence physique est devenue rare en prison de la part de l'administration pénitentiaire, une autre forme de violence a pris le relais. Une violence plus psychologique, plus tenace, plus insidieuse. Une violence qui ne dit pas son nom, qui a tout son temps. Celui des longues peines, celui des perpètes.

À la veille de l'élection présidentielle de 2002, un débat refait surface : celui de l'insécurité. On parle à nouveau de libérations "injustifiées ", de répression carcérale, de longueur des peines. Au milieu de ce brouhaha politico-médiatique, la question du sens de la prison, de l'opportunité des longues peines a du mal à se faire entendre. C'est pourtant bien de sens, et surtout de cohérence, que l'on doit parler, afin que la peine prononcée par un jury populaire ne soit pas déviée de son sens premier : punir certes, mais aussi réinsérer.

NicoLAS DE LAËK 


\section{Sociologie du temps carcéral et auto-analyse de la situation d'enquête en prison}

$\mathbf{L}$ a très belle enquête menée fera date dans l'abondante littérature sociologique sur la prison. On n'y trouvera pourtant pas de grandes conceptualisations sur l'univers carcéral, ni sur le temps ni sur le crime, pas plus sur l'identité et la préservation du moi face aux situations extrêmes. Comme toute très bonne recherche empirique, le lecteur s'imprègne de situations qui lui font très bien comprendre, par exemple, les notions de carcéralisme ou de résilience, sans lire de longs développements théoriques qui les enrobent. En déclinant les différents aspects du temps et en suivant les différentes phases par lesquelles les détenus condamnés passent durant leur très longue incarcération, l'auteure décrit des modalités particulières pour " rester au monde " au sein de l'univers carcéral, déjà si singulièrement hors du monde. Pour survivre en somme, malgré le " délit ", malgré l'enfermement, malgré l'absence de perspectives temporelles, malgré la vie qui continue en dehors...

Au temps discontinu et morcelé de la maison d'arrêt, incontrôlable puisque soumis aux aléas du procès, succède une phase d'adaptation à la situation de perpète - plus ou moins difficile selon les personnes, et parfois dramatique puisque se résolvant par le suicide. Le passage dans un établissement pour peine, au rythme totalement opposé à celui des maisons d'arrêt, est aussi un changement d'injonction : le détenu qui ne devait pas faire de vagues est maintenant sommé de se prendre en main. Il s'agit de "faire quelque chose de son temps ", alors que paradoxalement - mais la vie carcérale n'est traversée que de paradoxes - la politique d'aménagement des peines est en panne depuis près de dix ans. Pourtant, la sortie reste une perspective, parfois dramatique ( $c f$. les beaux passages sur l'angoisse de mourir en prison), mais qui reste fortement marquée par le poids du passé. Sur tous ces aspects, l'auteure a écrit un grand livre sur la politique pénale et le double langage de la politique pénitentiaire, qui démontre on ne peut mieux l'absurdité et la monstruosité des peines incompressibles.

"Monstruosité ", ce registre de vocabulaire qui revient si souvent chez ceux qui se réclament des victimes pour parler de leur bourreau, outre qu'il met définitivement hors jeu ceux qu'il accable, permet aussi d'éviter d'expliquer quoi que ce soit. Échapper au manichéisme : telle est la gageure de tout chercheur en prison. Sommé de choisir son camp, on assimile souvent le point de vue qu'il étudie (les perpètes ici, les surveillants pour d'autres) avec le camp qu'il devrait y défendre : pro-surveillant ou pro-détenu. Si l'auteure n'hésite pas à passer 
dans le registre de la dénonciation, quitte à outrepasser parfois la posture sociologique, elle n'en garde pas moins les distances nécessaires qui lui permettent de nous livrer un ouvrage extrêmement lucide. Bien sûr, certains développements auraient été enrichis par un croisement plus systématique des points de vue, mais plusieurs livres eurent été alors nécessaires. Surtout, AnneMarie Marchetti n'oublie pas qu'elle partage une commune humanité avec ses interlocuteurs et interlocutrices, auteurs de crimes souvent abominables, c'est-à-dire qu'elle restitue aussi ses craintes, ses angoisses, ses interrogations éthiques, son rapport singulier au crime (elle a elle-même été victime), etc. S'il fallait choisir, c'est sûrement cet aspect-là que je retiendrais : ce livre est un remarquable témoignage sur l'enquête dans un milieu difficile, qui implique une auto-analyse de la part du sociologue, particulièrement bien restituée ici, un modèle du genre à faire lire à tout apprenti enquêteur. On l'aura compris, ma voix dans cette rubrique où " plusieurs voix " divergent par leur statut vis-à-vis de l'objet (l'auteure, le perpète...) ne tarit pas d'éloge pour ce travail, qui mériterait donc d'être plus longuement discuté. Je me limiterai à trois aspects.

En approfondissant la problématique temporelle, on pourrait s'attarder un instant sur cette figure de la victime et la mémoire qu'incarne la peine en paiement du crime. Des trois fonctions attendues de la peine, préventive (futur), rétributive (passé) et réparatrice (présent), François Ost ${ }^{1}$ montre bien que le retour en force de la réparation correspond à celui de la victime qui prend maintenant une place centrale dans le procès pénal et dont

\section{Ce livre est un}

remarquable

témoignage sur

I'enquête dans un milieu

difficile, qui implique

une auto-analyse de

la part du sociologue,

particulièrement bien

restituée ici.

cette réparation se préoccupe essentiellement en compensant le dommage. Anne-Marie Marchetti attribue à la crise cette prééminence de la victime qui pousse toujours plus à la réclusion : les victimes du libéralisme triomphant s'identifient aux victimes de délits parce que celles-ci disposent d'un coupable visible. Mais on peut compléter cette explication en disant que la victime entretient un rapport d'immédiateté au procès, registre sur lequel procède la vengeance par exemple. Il faudrait donc peutêtre rapporter ce phénomène à celui plus général d'une forte tendance à la concentration du temps sur le présent (urgence, crise du projet, etc.), ce qui en l'occurrence vient contredire l'idée de justice.

Parmi les supports possibles pour survivre en tant que perpète (études, informatique, spiritualité...), les professionnels de la psychiatrie occupent une place particulière, de plus en plus importante. Anne-Marie Marchetti explique bien les ambivalences des détenus vis-àvis des psychiatres, eu égard notamment au rôle qu'ils jouent dans les expertises. Cependant, elle ne se concentre que sur cet aspect. Elle déplore à juste titre le manque patent d'intervenants, mais reste silencieuse sur l'implication des psychiatres soignants dans l'application des peines et sur le mythe de la prison réparatrice. Dans un contexte où la loi tend à aller de plus en plus vers des formes d'injonction de soins, n'y a-t-il pas à s'inquiéter du mélange des genres que l'on voit dans certaines pratiques, entre ordre carcéral, peine et soin ? Face à l'incarcération devenue quasi systématique des malades mentaux auteurs de délits, ne doiton pas rester plus attentif à l'assignation à la prison du rôle de dernier lieu de soins?

Enfin, les passages sur les valeurs sexuées (virilité, humanité...) qui traversent certaines relations ou pratiques en prison, notamment autour du corps, comme les soins, la musculation ou la sexualité, laissent poindre une tendance discutable à la naturalisation du social. La manière dont "l'homosexualité carcérale " n'est pensée essentiellement qu'en termes de sexualité de substitution en est un symptôme. Le recours aux entretiens, mobilisés dans ces développements, n'implique pas forcément de s'abstenir de déconstruire les catégories mobilisées. En ce sens, on aurait aimé voir plus systématiquement discutées les manières de résister à l'emprise carcérale, selon que l'on est dans une prison d'hommes ou de femmes.

Ce sont là quelques occasions de discussion qui ne doivent aucunement entacher le très grand respect qu'impose un tel travail.

MARC BESSIN

1. Le temps du droit, Odile Jacob, 1999. 